\title{
A NOTE ON LOCAL ASYMPTOTIC BEHAVIOR FOR BROWNIAN MOTION IN BANACH SPACES
}

\author{
MOU-HSIUNG CHANG \\ Department of Mathematics \\ The University of Alabama in Huntsville \\ Huntsville, Alabama 35807 \\ U.S.A.
}

(Received July 26, 1978)

ABSTRACT. In this paper we obtain an integral characterization of a two-sided upper function for Brownian motion in a real separable Banach space. This characterization generalizes that of Jain and Taylor [2] where $B=\mathbb{R}^{n}$. The integral test obtained involves the index of a mean zero Gaussian measure on the Banach space, which is due to Kuelbs [3] . The special case that when $B$ is itself a real separable Hilbert space is also illustrated.

KEY WORDS AND PHRASES. Gaussian measures on B-spaces, abstract wiener spaces, covariance operators, Brownian motion in B-space, upper and lower functions, integral test.

AMS(MOS) SUBJECT CLASSIFICATION (1970) CODES. Primary 60J65, 60617. 


\section{INTRODUCTION}

Let $B$ be a real separable Banach space with norm $\|\cdot\|$ and let $B$ * be the topological dual of $B$. If $\mu$ is a mean zero Gaussian measure on $B$ then it is well known from [1] that B contains a Hilbert space $H_{\mu}$ with norn $\|\cdot\| \|_{\mu}$ such that $\|\cdot\|$ is a measurable norm on $H_{\mu}$ in the sense of $[1]$. As a consequence, the $B$ norm $\|\cdot\|$ is weaker than $\|\cdot\|_{\mu}$. Thus through a restriction map we have the relation that $\mathrm{B}^{*} \subseteq \mathrm{H}^{*} \equiv \mathrm{H} \subseteq \mathrm{B}$. Furthermore, it is also shown in [1] that $\mu$ is the extension of the canonical normal distribution on $H_{\mu}$ to $B$ and we shall say that $\mu$ is generated by $\mathrm{H}_{\mu}$. If $\mathrm{K}$ denotes the unit ball of $\mathrm{H}_{\mu}$ in the norm $\|\cdot\| \|_{\mu}$, let $\Gamma=\sup _{x \in K}\|x\|$. The definition of index of $\mu, n_{1}$, is due to Kuelbs [3], where

$$
\begin{gathered}
n_{1}=\sup \left\{k: \exists f_{1}, \ldots, f_{k} \in B^{*} ; f_{1}, \ldots, f_{k} \text { orthogonal in } H_{\mu} ;\right. \\
\left.\left\|f_{j}\right\|_{B^{*}}=1 \text { and }\left\|f_{j}\right\|_{\mu}=\Gamma(1 \leq j \leq k)\right\} .
\end{gathered}
$$

It is known from [3] that $n_{1}$ exists and is finite and if $B$ itself is a Hilbert space then $n_{1}$ on $B$ equals the multiplicity of the maximal eigenvalue of the covariance operator for $\mu$. Let $\{W(t): 0 \leq t<\infty\}$ denote $\mu$-Brownian motion in $B$. Let $\Phi_{\varepsilon}$ denote the class of functions from $(0, \varepsilon)$ to $[0, \infty)$ such that $\phi(t) \uparrow \infty$ as $t \downarrow 0$ and $t^{1 / 2} \phi(t) \downarrow 0$ as $t \downarrow 0$.

DEFINITION 1. A function $\phi \in \Phi_{\varepsilon}$ is called an upper function for $\{W(t): t>0\}$ with respect to the norm $\|\cdot\|$, if given $t>0$, there exists a $\delta>0$ such that $P\left(|| W(t+v)-W(t-u)||<2^{1 / 2}(u+v)^{1 / 2} \phi(u+v) \Gamma\right.$ for all $u, v \geq 0$ with $0<\mathrm{u}+\mathrm{v}<\delta)=1$. In this case, we say $\phi \in U . \quad \phi \in \Phi_{\varepsilon}$ is called a lower function for $\{W(t): t \geq 0\}$ with respect to the norm $\|\cdot\|$, denoted by $\phi \in L$, if $\phi \notin U$. 
In the case that $B=\mathbb{R}^{d}$, a d-dimensional Euclidean space, Jain and Taylor [2] have shown that $\phi \in \Phi_{\varepsilon}$ is an upper function for d-dimension standard Brownian motion $\{W(t): 0 \leq t<\infty\}$ with respect to the Euclidean norm $\|\cdot\|_{2}$ if and only if ${ }_{0+} \frac{[\phi(t)]^{d+2}}{t} e^{-\phi^{2}(t) / 2 d t<\infty}$. This integral test for two-sided growth in $\mathbb{R}^{d}$ is the same as that for one-sided growth in $\mathbb{R}^{\mathrm{d}+2}$. In the case that $B$ is an infinite dimensional real separable Banach space, Kuelbs [3] has shown that $\phi(t)$, a nonnegative, non-decreasing continuous function defined for large values of $t$, is a one-sided upper function for $\mu$-Brownian motion $\{W(t): 0 \leq t<\infty\}$ with respect to some equivalent norm $\|\cdot\|_{1}$ on $B$ if and only if $J^{\infty} \frac{[\phi(t)]^{n_{1}}}{t} e^{-\phi^{2}(t) / 2} d t<\infty$ where the $\phi$ is called one-sided upper function with respect to $\|\cdot\| \|_{1}$ if $P\left(\|W(t)\|_{1}>t^{1 / 2} \phi(t) \Gamma\right.$ for only a bounded set of $\left.t^{\prime} s\right)=1$. Based on the results of Jain-Taylor and Kuelbs, it is very natural to conjecture that $\phi \in \Phi_{\varepsilon}$ is in $U$ (Definition 1) for $\{W(t): 0 \leq t<\infty\}$ with respect to some equivalent norm ||$\cdot \|_{1}$ on $B$ if and only if ${ }_{0+} \frac{[\phi(t)]^{n_{1}+2}}{t} e^{-\phi 2(t) / 2} d t<\infty$. The main purpose of this note is to verify this conjecture. Throughout this note $c$ will stand for a positive number whose value may change from line to line. The notation $a(h) \sim b(h)$ means $\lim _{h \rightarrow 0} \frac{a(h)}{b(h)}=1$.

2. MAIN RESULTS

The following useful estimates have been used repeatedly in [3] and they can be verified by the argument similar to that in d-dimensional Euclidean space $\mathbb{R}^{\mathrm{d}}[4$, p. 222].

LEMMA 2. Let $\{\mathrm{W}(\mathrm{t}): 0 \leq \mathrm{t}<\infty$ be Brownian motion in a real separable Banach space $\mathrm{B}$ having norm $\|\cdot\|$. Then for a11 $\lambda, \mathrm{h}>0$

$$
\begin{aligned}
& P\left(\sup _{0 \leq t_{1}<t_{2} \leq h}|| W(t)-W\left(t_{1}\right) \mid>\lambda h^{1 / 2}\right) \leq \\
& 2 P\left(\sup _{0 \leq t \leq h}|| W(t)||>\lambda h^{1 / 2}\right) \leq 4 P \quad\left(|| W(h)||>\lambda h^{1 / 2}\right) .
\end{aligned}
$$


We have the following integral test for a two-sided upper function for $\{W(t): 0 \leq t<\infty\}$ :

THEOREM 3. Let $\{W(t): 0 \leq t<\infty\}$ be $\mu$-Brownian motion in a real separable Banach space $B$ having norm $\|\cdot\|$, and assume $\phi \epsilon_{\varepsilon} \Phi_{\varepsilon}$ Let $\mathrm{n}_{1}$ denote the index of $\mu$. Then there is an equivalent norm $\|\cdot\|_{1}$ on $B$ such that $\sup _{x \in K}\|x\|_{1}=\Gamma$ and $\phi \varepsilon U$ with respect to $\|\cdot\|_{1}$ if and only if

$$
\int_{0+} \frac{[\phi(t)]^{n_{1}+2}}{t} e^{-\phi^{2}(t) / 2} d t<\infty .
$$

PROOF. The construction of the equivalent norm $\|\cdot\|_{1}$ is due to [3], which is defined to be

where

$$
\|\mathbf{x}\|_{1}=\max \left\{\Gamma|| \pi \mathbf{x}\left\|_{\mu},\right\| \mathrm{Qx} \|\right\}
$$

$$
\pi(x)=\sum_{j=1}^{n_{1}} e_{j}(x) e_{j}(x \in B)
$$

and

$$
Q(x)=x-\pi(x), e_{j}(\cdot) \text { denotes the linear function } f_{j}(\cdot) / \Gamma
$$

Consider the sequence $a_{m}=e^{-m / \log m}, m \geq 2$. Then $\frac{a_{m}}{a_{m+1}} \sim 1+(\log m)^{-1}$ as $m+\infty$. Let $u_{n, i}=\frac{i}{\log n} a_{n} ; v_{n, i}=\left(1-\frac{i}{\log n}\right) a_{n}, 0 \leq 1 \leq \log n$. Note that for each $i, u_{n, i}+v_{n, i}=a_{n}$. If $u, v \geq 0$ are sufficiently small we can choose an $n$ sufficiently large such that $a_{n+1} \leq u+v<a_{n}$. Assume that the integral (2) diverges. Define

$$
\begin{aligned}
& E_{n, i}=\left\{w:|| w\left(t+v_{n, i}\right)-w\left(t-u_{n, i}\right) \|_{1}>2^{1 / 2} a_{n}^{1 / 2} \phi\left(a_{n}\right) \Gamma\right\} \text { and } \\
& F_{n, i}=\left\{w:|| w\left(t+v_{n, i}\right)-w\left(t-u_{n, i}\right) \|_{\mu}>2^{1 / 2} a_{n}^{1 / 2} \phi\left(a_{n}\right)\right\} .
\end{aligned}
$$


Since

$\{\pi W(t): 0 \leq t<\infty\}$ is standard $n_{1}$ dimensional Brownian motion in $\pi B=\pi H_{\mu}$, divergence of the integral (2) implies that

$\sum_{n=2}^{\infty} \sum_{i=0}^{\log n} F\left(F_{n, i}\right)=\infty$ and infinitely many of $F_{n, i}$ occur.

Consequently infinitely many of $E_{n, i}$ occurr with probability one. Thus $\phi \varepsilon L$.

Now assume that the integral (2) converges. Let us choose a suitable

$i \leq \log n$ such that $u_{n, i} \leq u \leq u_{n, i+1}$ and $v \leq v_{n, i-1}$

Then

$$
\begin{aligned}
& P\left(|| W(t+v)-\left.W(t-u)\right|_{1}>2^{1 / 2}(u+v)^{1 / 2} \phi(u+v) \Gamma\right) \\
& \leq P\left(|| W(t+v)-\left.W(t-u)\right|_{1}>2^{1 / 2} a_{n+1}^{1 / 2} \phi\left(a_{n+1}\right) \Gamma\right) \\
& \leq P\left({ }_{0} \leq t_{1}<t_{2} \leq\left. u_{n, i+1} v_{n, i-1}|| \pi\left(W\left(t_{2}\right)-W\left(t_{1}\right)\right)\right|_{\mu}>2^{1 / 2} a_{n+1}^{1 / 2} \phi\left(a_{n+1}\right)\right) \\
& +P\left(\sup _{0} t_{1}<t_{2} \leq u_{n, i+1}+v_{n, i-1}|| Q\left(W\left(t_{2}\right)-W\left(t_{1}\right)\right) \mid>2^{1 / 2} a_{n+1}^{1 / 2} \phi\left(a_{n+1}\right) \Gamma\right) .
\end{aligned}
$$

Since $\{\pi W(t): 0 \leq t<\infty\}$ is standard $n_{1}$ dimensional Brownian motion in $\pi B=\pi H_{\mu}$, by the same argument as those in Theorem 3.1 [2], we conclude that the first term in the right hand side of the above inequality being zero for infinitely many $n$ and $i$. As for the second term in the above inequality, we have

$$
\begin{aligned}
& P\left({ }_{0 \leq t_{1}<t_{2} \leq u_{n, i+1}+v_{n, i-1}}|| Q\left(W\left(t_{2}\right)-W\left(t_{1}\right)\right)||>2^{1 / 2} a_{n+1}^{1 / 2} \phi\left(a_{n+1}\right) \Gamma\right) \equiv P\left(A_{n \cdot i}\right) \\
& \leq 4 P\left(|| Q W(1)||>\left(\frac{2 a_{n+1}}{u_{n, i+1}+v_{n, i-1}}\right)^{1 / 2} \phi\left(a_{n+1}\right) \Gamma\right) \\
& \leq C \exp \left\{-\varepsilon \frac{2 a_{n+1}}{\mu_{n, i+1}+v_{n, i-1}} \phi_{\left.\left(a_{n+1}\right) \Gamma^{2}\right\}}^{2}\right.
\end{aligned}
$$


Where

$$
\begin{aligned}
& \varepsilon<1 / 2 \text { sup }{ }_{B} \Lambda_{n}^{2}(x){ }_{\mu}^{Q}(d x),\left\{\Lambda_{j}\right\} \text { is a sequence in } B * \text { such that } \\
& \left\|\Lambda_{j}\right\|_{B^{*}}=1 \text { and }|| x||=\sup _{j}\left|\Lambda_{j}(x)\right| \text { for every } x \varepsilon B .
\end{aligned}
$$

The last inequality comes from [ 3, p. 253].

Since $u_{n, i+1}+v_{n, i-1}=(1+2 / \log n) a_{n} \sim(1+3 / \log n) a_{n+1}$, if

we choose $\delta$ be such that $\varepsilon=(1+\delta) / 2 \Gamma$ we have

$$
\begin{aligned}
P\left(A_{n, i}\right) & \leq C \exp \left\{-\delta \phi^{2}\left(a_{n+1}\right)\right\} \exp \left\{-\phi^{2}\left(a_{n+1}\right)\right\} \\
& \leq C \exp \left\{-\phi^{2}\left(a_{n+1}\right)\right\}
\end{aligned}
$$

Thus

$$
\begin{aligned}
\sum_{n=2}^{\infty} \sum_{i=0}^{\log n} P\left(A_{n, i}\right) & <c \sum_{n=2}^{\infty}(\log n) \exp \left\{-\phi^{2}\left(a_{n+1}\right)\right\} \\
& <c \sum_{n=2}^{\infty}\left[\phi\left(a_{n+1}\right)\right]^{n} 1 \exp \left\{-\phi^{2}\left(a_{n+1}\right)\right\} \\
& <\infty, \text { since the integral (2) converges }
\end{aligned}
$$

(see Lemma 2.12 of [2]).

From Lemma 2.15 (i) of [2] we conclude that $P\left(A_{n, i}, i .0.\right)=0$.

Thus

$$
P\left({ }_{t-u_{n, i+1} \leq t} \sup _{1}<t_{2} \leq t+v_{n, i-1}|| W\left(t_{2}\right)-\left.W\left(t_{1}\right)\right|_{1} \leq 2^{1 / 2} a_{n+1}^{1 / 2} \phi\left(a_{n+1}\right) \Gamma\right)=1
$$

for all $i$ and $n$ sufficiently large. Thus $\phi \varepsilon U$.

In case that $B$ is a real separable Hilbert space, then $n_{1}$ equals the multiplicity of the maximal eigenvalue of the covariance operator for $\mu$. We have the same result as those of Theorem 3 .

THEOREM 4. Let $\{W(t): 0 \leq t<\infty\}$ be $\mu$-Brownian motion in a real separable Hilbert space $H$ with norm $\|\cdot\|$, and suppose $\phi \in \Phi_{\varepsilon}$. Then $\phi$ 
is in $U$ with respect to the given norm $\|\cdot\|$ if and only if

$$
s_{0+} \frac{[\phi(t)]^{n_{1}+2}}{t} e^{-\phi^{2}(t) / 2} d t<\infty
$$

Where $n_{1}$ denotes the multiplicity of the maximal eigenvalue of the covariance operator for $\mu$.

PROOF. Let sequence $\left\{a_{n}\right\},\left\{u_{n, i}\right\}$ and $\left\{v_{n, 1}\right\}$ be the same as those in the proof of Theorem 3. If $u, v \geq 0$ are sufficiently small we choose n sufficiently large such that $a_{n+1} \leq u+v<a_{n}$ and then $f i x i \leq \log n$ such that $u_{n, i} \leq \mathrm{u} \leq \mathrm{u}_{\mathrm{n}, i+1}$ and $\mathrm{v} \leq \mathrm{v}_{\mathrm{n}, \mathrm{i}-1}$. If the integral (3) diverges, then we proceed as those in Theorem 3 and conclude that $\phi \in L$. Now if the integral (3) converges define

$$
B_{n, i}=\left\{w: \quad t-u_{n, i+1} \leq t_{1}<t_{2} \leq t+v_{n, i}|| w\left(t_{2}\right)-w\left(t_{1}\right)||>2^{1 / 2} a_{n+1}^{1 / 2} \phi\left(a_{n+1}\right) \Gamma\right\} .
$$

Then

$$
\begin{aligned}
P\left(B_{n, i}\right) & \leq 4 P\left(|| W(1)||>\left(\frac{2 a_{n+1}}{u_{n, i+1} v_{n, i-1}}\right)^{1 / 2} \phi\left(a_{n+1}\right) \Gamma\right) \\
& \leq C\left[\phi\left(a_{n+1}\right)\right]^{n_{1}^{2}} \exp \left(-\frac{a_{n+1} \phi^{2}\left(a_{n+1}\right)^{\Gamma^{2}}}{\left(u_{n, i+1}+v_{n, i-1}\right) \lambda}\right) \\
& \leq C\left[\phi\left(a_{n+1}\right)\right]^{n_{1}^{-2}} \exp \left\{-\phi^{2}\left(a_{n+1}\right)\right\}
\end{aligned}
$$

Where $\lambda$ is the maximal eigenvalue of the covarience of $\mu$ and it is known that $\lambda=\Gamma^{2}$ [3]. The last inequality comes from [3] and the fact that $u_{n, i+1}+v_{n, i-1} \sim \frac{a_{n+1}}{1+3(\log n)^{-1}}$

Thus

$$
\begin{aligned}
\sum_{n=2}^{\infty} \sum_{i=0}^{\log n} P\left(B_{n, i}\right) & <C \sum_{n=2}^{\infty} \sum_{i=0}^{\log n}\left[\phi\left(a_{n+1}\right)\right]^{n_{1}-2} \exp \left\{-\phi^{2}\left(a_{n+1}\right)\right\} \\
= & C \sum_{n=2}^{\infty}\left[\phi\left(a_{n+1}\right)\right]^{n_{1}} \exp \left\{-\phi^{2}\left(a_{n+1}\right)\right\}<\infty \text { since }
\end{aligned}
$$

the integral (3) converges. From Lemma 2.15 (i) we have $P\left(B_{n, i}, i .0.\right)=0$. 
That is $P\left(B_{n, i}^{c}\right)=1$ for sufficiently large $i$ and $n$. Thus $\phi \in U$.

\section{REFERENCES}

1. Gross, L. Abstract Wiener Spaces, Proc. Fifth Berkeley Symposium on Math Statistics and Probability, (1965) 31-42.

2. Jain, N. C. and Taylor, S. J. Local Asymptotic Laws for Brownian Motion. The Annals of Probability, Vol. 1, No. 4 (1973) 527-549.

3. Kuelks, J. Sample Path Behavior for Brownian Motion in Banach Spaces. The Annals of Probability, Vol. 3, No. 2 (1975) 247-261.

4. Taylor, S. J. Exact Asymptotic Estimates of Brownian Path Variation. Duke Math. J. 39, (1972) 219-241. 


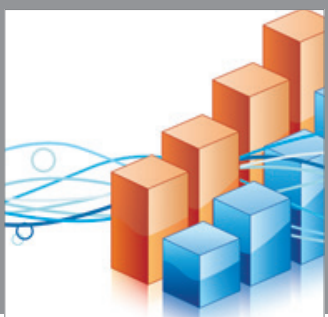

Advances in

Operations Research

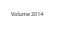

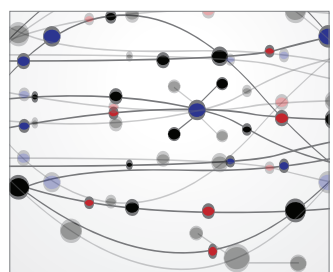

\section{The Scientific} World Journal
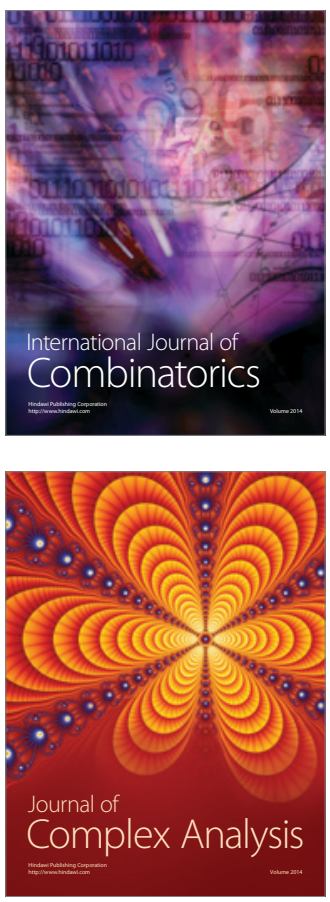

International Journal of

Mathematics and

Mathematical

Sciences
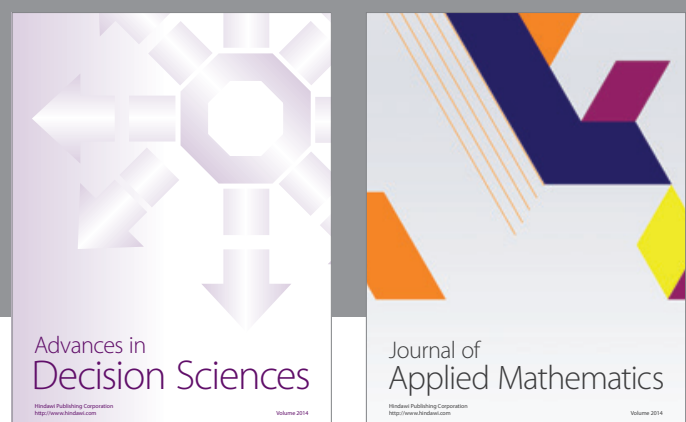

Journal of

Applied Mathematics
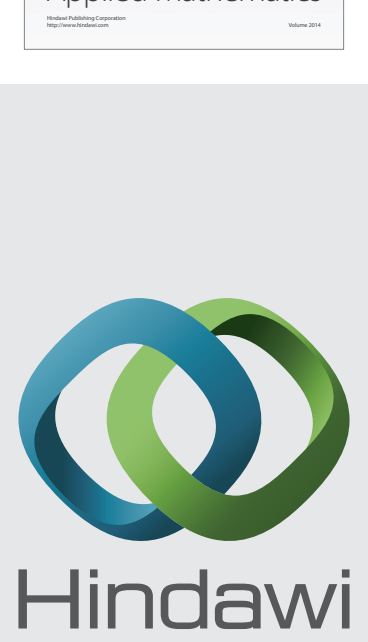

Submit your manuscripts at http://www.hindawi.com
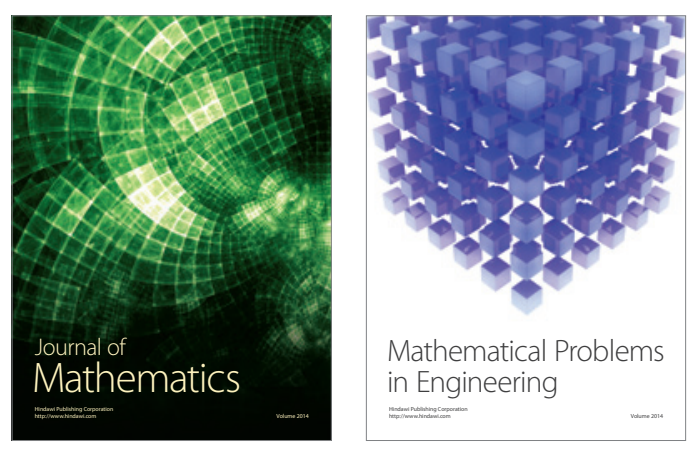

Mathematical Problems in Engineering
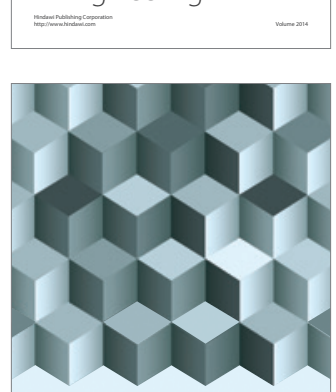

Journal of

Function Spaces
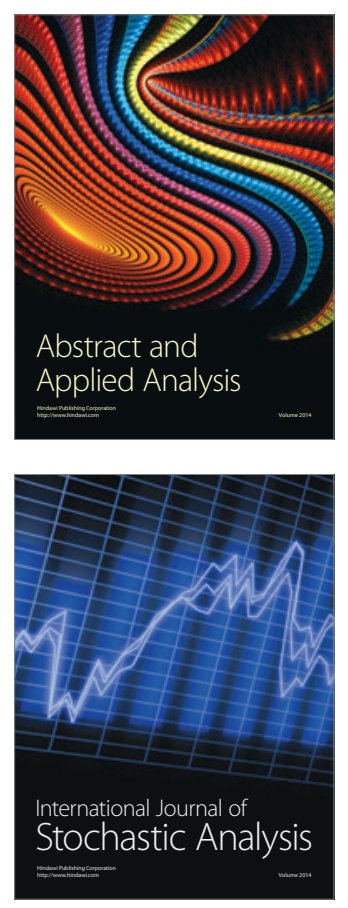

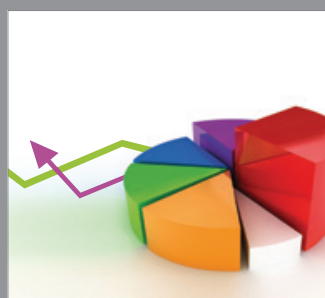

ournal of

Probability and Statistics

Promensencen
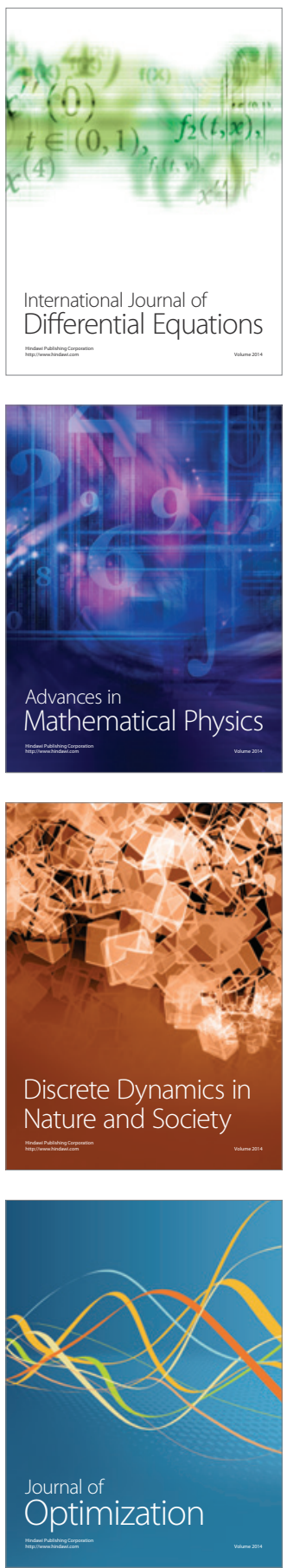